\title{
Hexaferrite-FeCo nanocomposite particles and their electrical and magnetic properties at high frequencies
}

\author{
C. Sudakar, G. N. Subbanna, ${ }^{\text {a) }}$ and T. R. N. Kutty ${ }^{\text {b) }}$ \\ Materials Research Centre, Indian Institute of Science, Bangalore 560012, India
}

\begin{abstract}
Nanocomposites are realized by chemical reduction whereby the conducting magnetic particles of $\mathrm{Fe}-\mathrm{Co}$ alloy are generated within the insulating ferrimagnetic $\mathrm{BaCo}_{2} \mathrm{Fe}_{16} \mathrm{O}_{27}$ or $\mathrm{Ba}_{2} \mathrm{Co}_{2} \mathrm{Fe}_{12} \mathrm{O}_{22}$ hexaferrite matrix. Transmission electron microscopy revealed that metal nanoparticles precipitate coherently as thin flakes along the $a-b$ planes of the derivative magnetoplumbite lattice of the hexaferrites above the characteristic reduction temperature, $T_{R}>375^{\circ} \mathrm{C}$ in $\mathrm{H}_{2}$ atmosphere. The coercivity increases with $T_{R}$ in the early stages of the solid-state precipitation and then decreases with the formation of larger fractions of $\mathrm{Fe}-\mathrm{Co}$ alloy; a converse trend is noticed for magnetization. The complex permittivity increases with reduction to $\sim 50$ in the broad frequency range of 4-18 $\mathrm{GHz}$. The complex permeability is also enhanced with the content of $\mathrm{Fe}-\mathrm{Co}$ nanoparticles. It is proposed that the spin reorientation at the $\mathrm{Fe}-\mathrm{Co} / \mathrm{hexaferrite}$ interface gives rise to broadband response, rendering these composite particles useful as electromagnetic microwave absorbers.
\end{abstract}

\section{INTRODUCTION}

Composites, where the magnetic particles are usually embedded in either insulating or conducting matrix, are often employed in high speed electronic circuits to reduce the electromagnetic interference (EMI), decrease the noise level of signals and ensure the electromagnetic compatibility. ${ }^{1} \mathrm{EM}$ radiation shields, either reflection or absorption based, need be thin and lightweight, responsive to broader frequency range and nearly independent of the incident angle. As far as the thickness and working frequency bandwidth are concerned, the magnetic composites have definitive advantages over their dielectric analogues. The most commonly used fillers are spinel ferrites or hexaferrites. The latter with planar magnetic anisotropy are of great interest for use as electromagnetic energy dissipation materials in microwave frequency range. ${ }^{2} \mathrm{BaCo}_{2} \mathrm{Fe}_{16} \mathrm{O}_{27}\left(\mathrm{WCo}_{2}\right)$ and $\mathrm{Ba}_{2} \mathrm{Co}_{2} \mathrm{Fe}_{12} \mathrm{O}_{22}$ $\left(\mathrm{YCo}_{2}\right)$ hexaferrites are soft magnetic materials with planar anisotropy; they have relatively high resonance frequency and high permeability. However for broadband operation, magnetic nanocomposites are desirable. Nanosize iron particles dispersed in an oxide matrix by reaction milling have been studied. ${ }^{3}$ However composite materials with conducting magnetic particles generated in situ by chemical reactions within the ferrimagnetic hexaferrite insulating matrix are scarcely known.

\section{EXPERIMENT}

In this article we report the structural, magnetic, and microwave absorption properties at very high frequencies $(4-18 \mathrm{GHz})$ of the hexaferrite-FeCo alloy nanocomposites obtained by controlled chemical reduction of hexa-

\footnotetext{
a) Deceased on 16th July 2003.

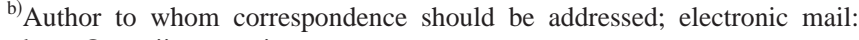
kutty@mrc.iisc.ernet.in
}

ferrite, $\mathrm{WCO}_{2}$, and $\mathrm{YCo}_{2} . \mathrm{WCo}_{2}$ and $\mathrm{YCo}_{2}$ hexaferrites with oriented grains were prepared by heat-treating at $\sim 1250{ }^{\circ} \mathrm{C}$ the precursors obtained by the wet chemical gel-to-crystallite conversion. ${ }^{4}$ Hexaferrite particles on controlled reduction in flowing hydrogen at $350-450{ }^{\circ} \mathrm{C}$ causes in situ precipitation of FeCo alloy (iron rich) nanoparticles within the hexaferrite matrix. Sample identifications used in the subsequent sections have been denoted with the type of hexaferrite followed by the heat-treatment condition in $\mathrm{H}_{2}$. Thus, for example, $\mathrm{WCo}_{2}-\mathrm{R} 400$ represents $\mathrm{WCo}_{2}$ hexaferrite reduced at $400{ }^{\circ} \mathrm{C}$. Phase identification of the powders was investigated by x-ray diffraction (XRD) using a Philips PW 1050 diffractometer equipped with $\mathrm{Cu} K \alpha$ radiation. Metal content in the composite is evaluated by wet chemical analyses ${ }^{5}$ as well by comparing the relative intensity of $\mathrm{x}$-ray reflections of hexaferrite and iron from standard mixtures. Further, the compositional variations are evaluated by $\mathrm{x}$-ray photoelectron spectra (XPS) of the samples with a VG ESCA 3 MK II instrument using $\mathrm{Al} K \alpha$ x rays. Transmission electron microscopy observations were performed on the nanocomposites for morphological and lattice imaging studies, with a JEOL, JEM 200CX microscope. Magnetization measurements were carried out between -9 and $+9 \mathrm{kOe}$ at room temperature, by means of a vibrating sample magnetometer (VSM) (Lakeshore 7300). Complex permittivities $\left(\varepsilon^{\prime}-j \varepsilon^{\prime \prime}\right)$ and complex permeabilities $\left(\mu^{\prime}-j \mu^{\prime \prime}\right)$ were calculated from the measured reflection coefficients using the vector network analyzer (Agilent $8722 \mathrm{ES}$ ) in the frequency range of 4-18 $\mathrm{GHz}$, wherein the samples are loaded in coaxial cells.

\section{RESULTS AND DISCUSSION}

Figure 1 shows the XRD patterns of $\mathrm{WCo}_{2}$ and $\mathrm{YCo}_{2}$ hexaferrites heat treated at $1250{ }^{\circ} \mathrm{C}$. The relative intensities of $\mathrm{WCo}_{2} \mathrm{x}$-ray reflections show oriented grain growth along certain crystallographic planes, predominantly $(00 l)$ reflec- 


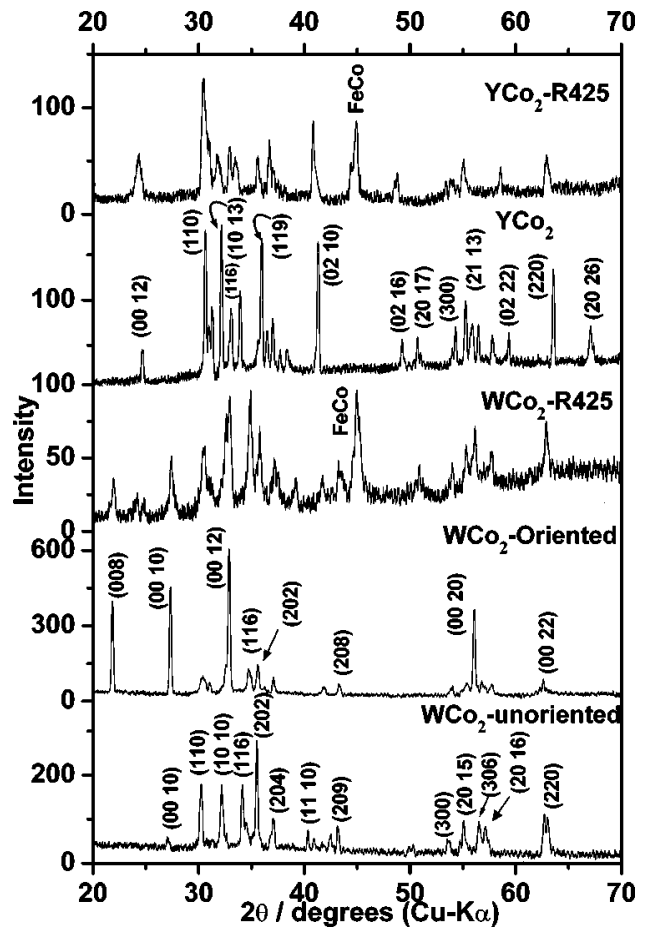

FIG. 1. XRD patterns of hexaferrites $\left(\mathrm{WCO}_{2}\right.$ and $\left.\mathrm{YCo}_{2}\right)$ before and after reduction.

tions and $(h k l)$ with larger $l(>h+k)$ as strong peaks, indicating that the particles are grown as platelets with the $c$ axis nearly perpendicular to the plane of observation. The average grain size of the particles from scanning electron microscopy is $\sim 20 \mu \mathrm{m}$. The XRD patterns of $\mathrm{YCo}_{2}$ also show narrow linewidths indicative of high crystallinity. On reducing these crystallites chemically at $350-450{ }^{\circ} \mathrm{C}$ in hydrogen atmosphere, reflections of $\mathrm{Fe}-\mathrm{Co}$ alloy (bcc) show up in the XRD patterns along with those of hexaferrite. However, reflections of the latter become line broadened and the intensities decrease drastically with the extent of reduction. At $T_{R}$ $\geqslant 450{ }^{\circ} \mathrm{C}$, the intensities of $\mathrm{Fe}-\mathrm{Co}$ reflections increase. The characteristic temperature at which discernible reduction takes place, as observed from XRD and chemical analyses, differs for $\mathrm{WCo}_{2}$ and $\mathrm{YCo}_{2}$. $\mathrm{WCo}_{2}$ has $\sim 5 \%$ FeCo forma-

TABLE I. Estimated $\%$ of FeCo at different $T_{R}$ and magnetic properties of $\mathrm{WCO}_{2}$ and $\mathrm{YCo}_{2}$ hexaferrites.

\begin{tabular}{|c|c|c|c|c|}
\hline $\begin{array}{l}\text { Sample } \\
\text { Identification }\end{array}$ & $\begin{array}{c}T_{R} \\
\left({ }^{\circ} \mathrm{C}\right)\end{array}$ & $\begin{array}{l}\% \text { of } \mathrm{FeCo} \\
\text { (Fe/Co from } \\
\text { XPS } \\
\text { analyses) }\end{array}$ & $\begin{array}{l}M_{S} \\
(\mathrm{emu} / \mathrm{g})\end{array}$ & $\begin{array}{l}H_{C} \\
(\mathrm{Oe})\end{array}$ \\
\hline $\mathrm{WCo}_{2}-\mathrm{R} 375$ & 375 & ----- & 69.7 & 200 \\
\hline $\mathrm{WCo}_{2}-\mathrm{R} 400$ & 400 & 4.9 & 62 & 247 \\
\hline $\mathrm{WCo}_{2}-\mathrm{R} 425$ & 425 & $12.6(7.5)$ & 53 & 496 \\
\hline $\mathrm{WCo}_{2}-\mathrm{R} 450$ & 450 & $17.8(7.1)$ & 50.5 & 581 \\
\hline $\mathrm{WCo}_{2}-\mathrm{R} 500$ & 500 & $35(6.5)$ & 69 & 645 \\
\hline $\mathrm{WCo}_{2}-\mathrm{R} 700$ & 700 & $75(6.6)$ & 169.7 & 52 \\
\hline $\mathrm{YCo}_{2}-\mathrm{R} 400$ & 400 & ----- & 35 & 205 \\
\hline $\mathrm{YCo}_{2}-\mathrm{R} 425$ & 425 & 5.2 & 32.4 & 275 \\
\hline $\mathrm{YCo}_{2}-\mathrm{R} 450$ & 450 & $14(5.2)$ & 30.8 & 437 \\
\hline $\mathrm{YCo}_{2}-\mathrm{R} 500$ & 500 & 25 (4.6) & 38.6 & 694 \\
\hline $\mathrm{YCo}_{2}-\mathrm{R} 700$ & 700 & $54(4.6)$ & 122.6 & 72 \\
\hline
\end{tabular}
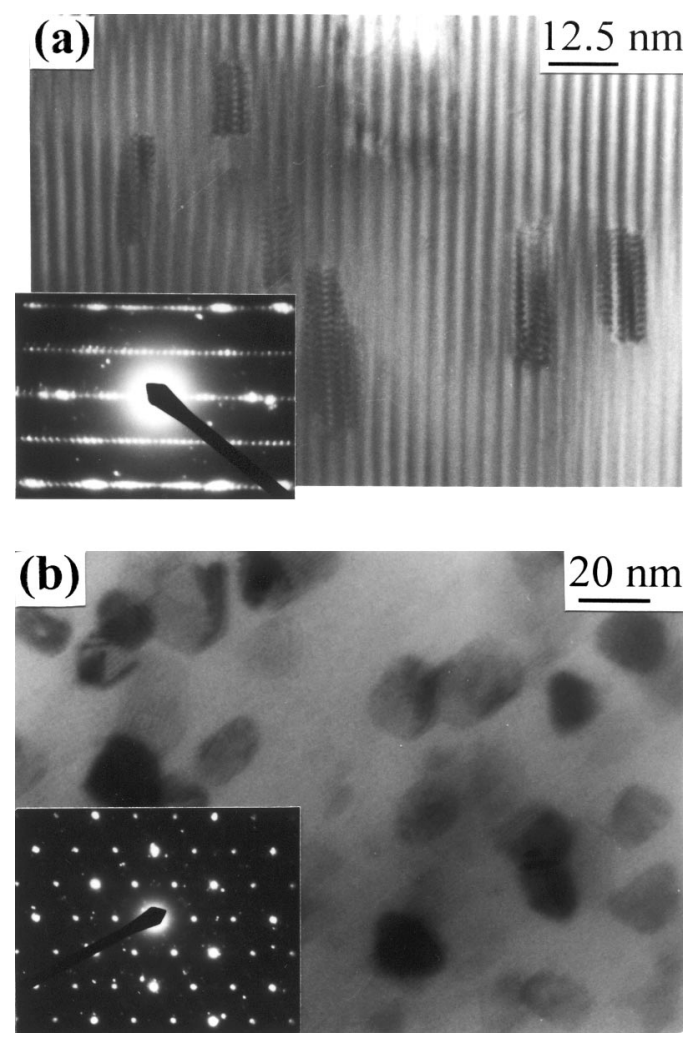

FIG. 2. High resolution electron micrographs of the $\mathrm{WCo}_{2}-\mathrm{R} 425$ nanocomposite with beam direction along (a) [110] and (b) [001]. Corresponding SAED patterns are shown in the insets.

tion at $T_{R} \cong 400{ }^{\circ} \mathrm{C}\left(\mathrm{WCo}_{2}-\mathrm{R} 400\right)$ for $1 \mathrm{~h}$, whereas $\mathrm{YCo}_{2}$ has the same percent of reduction at $T_{R} \sim 425^{\circ} \mathrm{C}$ $\left(\mathrm{YCo}_{2}-\mathrm{R} 425\right)$. Above these temperatures, the percentage of $\mathrm{Fe}-\mathrm{Co}$ increases exponentially. The estimated ( $\mathrm{Fe}-\mathrm{Co})$ content for samples heat treated at different temperatures in hydrogen atmosphere is given in Table I. The atomic ratio of Fe and Co estimated from XPS analyses in the reduced samples of $\mathrm{WCO}_{2}$ is in the range of $6-8$, whereas in $\mathrm{YCo}_{2}$ it is around 4-6.

Figure 2(a) shows the high resolution electron microscope image of the oriented hexaferrite particles $\left(\mathrm{WCo}_{2}\right)$ reduced at $425^{\circ} \mathrm{C}$. Uniform lattice fringes are discernible with regular coherent growth of the basic structural blocks in $\mathrm{WCo}_{2}$, which are disturbed with the extent of chemical reduction. Just near the characteristic reaction temperature, $T_{R} \sim 400^{\circ} \mathrm{C}$, the lattice images reveal embedded metal particles coherently growing along the $a-b$ planes. The metallic region expands with higher $T_{R}\left(\geqslant 425^{\circ} \mathrm{C}\right)$. The lattice gets distorted as can be deciphered from the wrinkling of the lattice fringes due to stacking faults. This leads to further disintegration of the monocrystalline particles at $T_{R}$ $\geqslant 450{ }^{\circ} \mathrm{C}$ to composite multicrystallites of separate phases ( $\mathrm{Fe}-\mathrm{Co}$ alloy, $\mathrm{BaO}$, and $\mathrm{BaFe}_{2} \mathrm{O}_{4}$ ). Interestingly, the reduced regions grow perpendicular to the $c$ axis (along $a-b$ plane) of hexagonal magnetoplumbite structure. The high resolution image of a particle taken with the beam along the $c$ axis reveals the internal structure of hexaferrite platelet with the embedded metal particle as thin nanoflakes [Fig. 2(b)]. The particles are well separated at $T_{R} \leqslant 425^{\circ} \mathrm{C}$. The selected area 


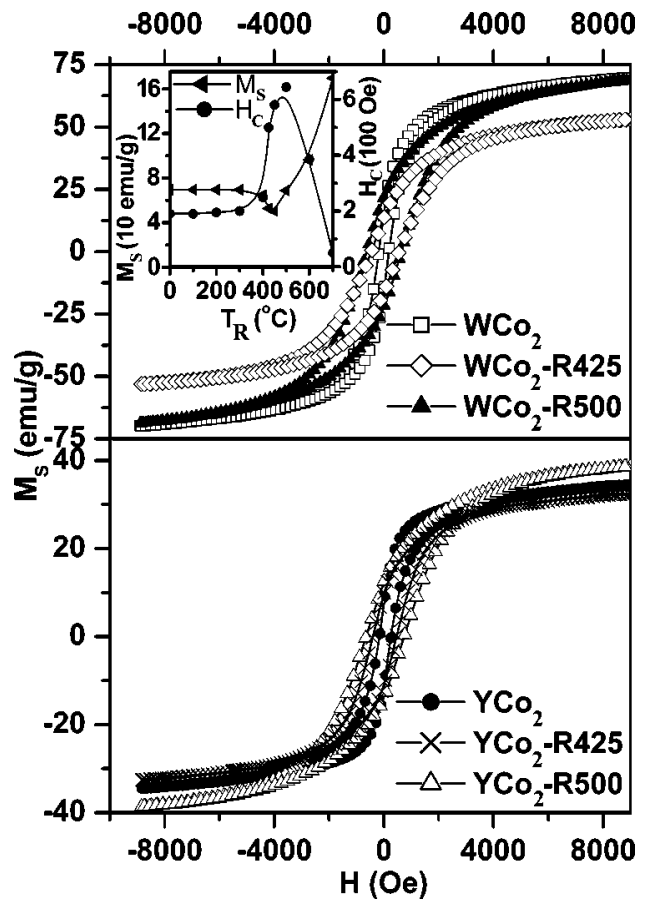

FIG. 3. $M_{S}-H$ curves of $\mathrm{WCo}_{2}$ and $\mathrm{YCo}_{2}$ samples reduced at different $T_{R}$. Inset shows the variation of $M_{S}$ and $H_{C}$ with $T_{R}$ for reduced $\mathrm{WCo}_{2}$ hexaferrites.

electron diffraction (SAED) of such composites show very feeble reflections from the embedded metal nanoparticles. Similar observations were made with $\mathrm{Y}-\mathrm{Co}_{2}$ hexaferrite as well. However, the metal particles grow and coalesce, followed by the disintegration of the matrix at higher $T_{R}$.

The particles with composite characteristics, namely the hexaferrite matrix still remaining intact with minimum defects and the coherent flaky metallic phase (FeCo alloy) precipitated in situ, give rise to modified magnetic properties as well as microwave absorption in contrast to the corresponding bulk phases. The variation of magnetization as a function of applied magnetic field for differently reduced samples is shown in Fig. 3. For $\mathrm{W}-\mathrm{Co}_{2}$ hexaferrite, the as-prepared particles have specific magnetization, $M_{S}=69.7 \mathrm{emu} / \mathrm{g}$ and coercivity, $H_{C}=190 \mathrm{Oe}$, whereas samples reduced at $400{ }^{\circ} \mathrm{C}$ in $\mathrm{H}_{2}$ have $M_{S} \approx 62 \mathrm{emu} / \mathrm{g}$ and $H_{C} \approx 250$ Oe. For $\mathrm{WCo}_{2}-\mathrm{R} 425 M_{S}$ decreases further to $\sim 53 \mathrm{emu} / \mathrm{g}$, whereas $H_{C}$ increases to $\sim 500 \mathrm{Oe}$. The decrease in $M_{S}$ can be understood from the formation of defective hexaferrite matrix with nanocrystalline $\mathrm{Fe}-\mathrm{Co}$ particles. On the other hand, the coercivity of the particles increased due to the pinning of the domains by the presence of defects. Thus, the composites exhibit combination dependent magnetic property rather than contributions from the individual components. For samples reduced at $500{ }^{\circ} \mathrm{C}, M_{S}$ increases to $69 \mathrm{emu} / \mathrm{g}$ with $H_{C}$ further increasing to $645 \mathrm{Oe}$. The increase in $M_{S}$ results from the larger amounts of coarser $\mathrm{Fe}-\mathrm{Co}$ alloy formation. The increasing trend of coercivity indicates that the defects still play a role in pinning down the domain reversals in the ferrimagnetic components. The trend of changes in $M_{S}$ and $H_{C}$ with the $T_{R}$ is shown clearly in the inset of Fig. 3. For deeply reduced samples (at $\sim 700^{\circ} \mathrm{C}$ ) the magnetization increases
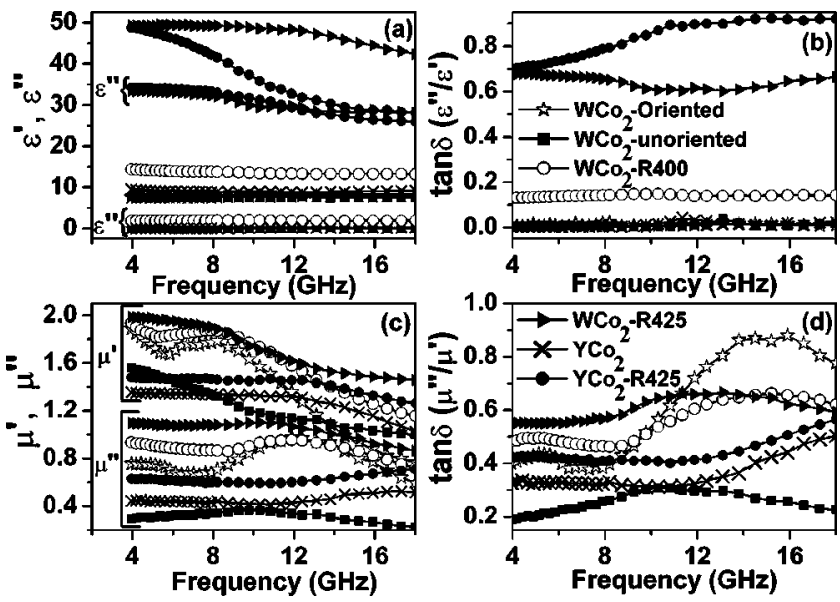

FIG. 4. Frequency dependence of (a) $\varepsilon^{\prime}$ and $\varepsilon^{\prime \prime}$, (b) $\tan \delta\left(\varepsilon^{\prime \prime} / \varepsilon^{\prime}\right)$, (c) $\mu^{\prime}$ and $\mu^{\prime \prime}$, and (d) $\tan \delta\left(\mu^{\prime \prime} / \mu^{\prime}\right)$ of $\mathrm{WCo}_{2}$ and $\mathrm{YCo}_{2}$ before and after reduction.

enormously $(\sim 169 \mathrm{emu} / \mathrm{g})$, indicating $\sim 75 \%$ (by weight) constitute the metallic FeCo particle. $\mathrm{Y}-\mathrm{Co}_{2}$ hexaferrites reduced in $\mathrm{H}_{2}$ display nearly similar changes (Fig. 3). The magnetic properties of reduced samples of $\mathrm{YCo}_{2}$ hexaferrites are listed in Table I. The as-prepared samples of $\mathrm{Y}-\mathrm{Co}_{2}$ have $M_{S}=35 \mathrm{emu} / \mathrm{g}$ and $H_{C}=205 \mathrm{Oe}$. The coercivity initially increases with the $T_{R}\left(<500{ }^{\circ} \mathrm{C}\right)$ and then decreases with the precipitation of more $\mathrm{Fe}-\mathrm{Co}$ in the composite. Magnetization decreases when $T_{R} \leqslant 450{ }^{\circ} \mathrm{C}$, with a reversing trend when the samples are reduced at higher temperatures.

The complex dielectric as well as magnetic properties of the ferrite powders are measured in the region of $4-18 \mathrm{GHz}$. The dielectric properties of the samples do not show significant changes in comparison to the bulk at these frequency ranges [Figs. 4(a) and 4(b)]. However, the properties differ for the hexaferrites after reduction. The as-prepared powders have real part of complex permittivity $\left(\varepsilon^{\prime}\right)$ around $7-8$ and the imaginary parts $\left(\varepsilon^{\prime \prime}\right)$ of $0.1-0.3$ for $\mathrm{WCo}_{2}$ and $\mathrm{YCo}_{2}$. The $\varepsilon^{\prime}$ increases with the extent of reduction. For $\mathrm{WCo}_{2}-\mathrm{R} 400, \varepsilon^{\prime}$ increases to $\sim 12$ and further to $\sim 50$ for $\mathrm{WCo}_{2}-\mathrm{R} 425$. $\varepsilon^{\prime}$ decreases with increasing frequency for $\mathrm{WCo}_{2}-\mathrm{R} 425$ and $\mathrm{YCo}_{2}-\mathrm{R} 425$. The imaginary part of permittivity $\left(\varepsilon^{\prime \prime}\right)$ also shows an increase to $\sim 30$ for these samples. For the as-prepared samples, the dielectric loss tangent $\left(\tan \delta=\varepsilon^{\prime \prime} / \varepsilon^{\prime}\right)$ is very small with near zero values, whereas, $\tan \delta$ is high for reduced samples. The real $\left(\mu^{\prime}\right)$ and imaginary $\left(\mu^{\prime \prime}\right)$ parts of relative complex permeability are shown in Figs. 4(c) and 4(d), wherein $\mu^{\prime}$ decreases with frequency in all samples owing to the broadening of ferrimagnetic resonance. The magnetic resonance behavior is better discernible in oriented $\mathrm{WCo}_{2}$ and $\mathrm{YCo}_{2}$ grains. The natural resonance frequency due to spin rotation is around 12-13 $\mathrm{GHz}$ for $\mathrm{WCo}_{2}$ and $\sim 18 \mathrm{GHz}$ for $\mathrm{YCo}_{2}$, corresponding to the maximum observed in $\mu^{\prime \prime}$ frequency curves. These samples have larger values of magnetic loss tangent $(\tan \delta$ $\left.=\mu^{\prime \prime} / \mu^{\prime}\right)$ in the vicinity of the resonance frequency, whereas for unoriented crystals, $\mu^{\prime \prime}$ is low $(\sim 0.3)$ with shallow magnetic loss and the resonance is discernible around 8-10 GHz. The magnetic loss spectra have similar changes with $\mu^{\prime \prime}$ and have the highest absorption loss just above the resonance frequency, where the loss tangent is maximum. The maxi- 
mum magnetic loss is observed for oriented $\mathrm{W}-\mathrm{Co}_{2}$ hexaferrite at $\sim 15 \mathrm{GHz}$. For reduced samples, permeability increases slightly with the reduction temperature. The larger permeability in the high frequency region for the composite particles in which the flaky thin metal nanoparticles are oriented in the $a-b$ planes of the hexagonal platelets, results from the anisotropic morphology of the constituent particles. Thus, the hexaferrite metal nanoparticle composites show considerably smaller reluctance in the oriented directions than the unoriented particle system. The high permeability of the magnetic composite over a broad range of frequency (4-18 GHz) makes it possible to produce thinner electromagnetic wave absorbers. The magnetic structure at the metal/hexaferrite interface in the composite, with magnetization direction dictated by the combined anisotropy fields of the ferrimagnetic components, can lead to its own characteristic relaxation processes due to complex spin reorientation at high frequencies. Thus the magnetic loss spectra show broad absorption from 4 to $18 \mathrm{GHz}$.

\section{CONCLUSION}

In conclusion, composites of hexaferrites containing $\mathrm{Fe}-\mathrm{Co}$ alloy (iron rich) nanoparticles precipitated in situ are good candidates for wide bandwidth electromagnetic microwave absorption in broad frequency ranges. Such nanocomposites are easier to prepare when compared to iron particle dispersed in oxide matrix by mechanochemical milling.

\section{ACKNOWLEDGMENT}

The Board for Smart Materials Research and Technology of NPSM, Government of India, is thanked for the research funding.

${ }^{1}$ S.-H. Yu and M. Yoshimura, Adv. Funct. Mater. 12, 9 (2002).

${ }^{2}$ T. Inui, K. Konishi, and K. Oda, IEEE Trans. Magn. 35, 3148 (1999).

${ }^{3}$ M. Pardavi-Horvath, J. Magn. Magn. Mater. 215-216, 171 (2000).

${ }^{4}$ C. Sudakar, G. N. Subbanna, and T. R. N. Kutty, J. Magn. Magn. Mater. 263, 253 (2003).

${ }^{5}$ A. I. Vogel, Textbook of Quantitative Chemical Analysis, 5th ed. (Longman, Singapore, 1991), p. 376. 